\title{
Factors Affecting Concordance between Radiological and Histological Findings in Invasive Lobular Carcinoma - Experience from a National Cancer Centre
}

\author{
Duaa Abu-Sinn $^{\mathrm{a}} \quad$ Donal O’Driscoll $^{\mathrm{b}}$ Maurice Murphy ${ }^{\mathrm{a}}$

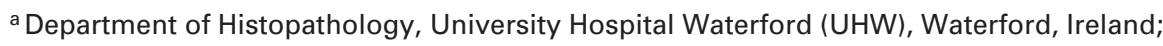 \\ ${ }^{b}$ Department of Radiology, University Hospital Waterford (UHW), Waterford, Ireland
}

\section{Keywords}

Invasive lobular carcinoma - Radiology-histology concordance - Pattern of invasion - Biological factors .

Biomarker · Breast cancer - Disease management

\section{Summary}

Background: Invasive lobular carcinoma (ILC) is characterized by an infiltrative discohesive growth pattern, making it difficult to accurately assess both clinically and by imaging studies. Despite favourable biological characteristics, challenges remain in the surgical treatment of ILC. We aimed to evaluate radiology/histology concordance and identify histological and biological parameters on core biopsies that may predict final tumour histology and guide surgical treatment decisions. Patients and Methods: The radiology and histology reports for all newly diagnosed cases of ILC were analysed. The biopsy and resection histological slides for all the surgical cases were reviewed. Results: 75 new cases of ILC were diagnosed over a 2-year period. 48 patients underwent surgery of whom $25 \%$ had 2 or more operations. There was discordance between radiological and histological tumour focality and tumour size in 35 and $40 \%$, respectively. The correlation between radiology/histology discordance and E-cadherin expression was statistically significant. However, the correlation between radiology/ histology discordance and menopausal status, breast density, pattern of invasion, presence of lobular intraepithelial neoplasia (LIN), hormonal status, and Ki67 were not statistically significant. Conclusion: Histological and biological factors in ILC, with the exception of E-cadherin expression, do not seem to play a significant role in radiology/histology discordance. However, larger studies are needed to further corroborate these findings.

(C) 2017 S. Karger GmbH, Freiburg

\section{Introduction}

Invasive lobular carcinoma (ILC) of the breast is the most common 'special' morphological subtype of breast cancer, comprising up to $15 \%$ of all cases. The cardinal feature of ILC is their inherently discohesive phenotype largely attributable to E-cadherin loss. There are 4 recognizable histological patterns of ILC: classical, trabecular, solid, and alveolar. In classical ILC, the characteristic pattern of growth involves the infiltration of single cells or single files of cells through the stroma, with little disturbance of the normal tissue architecture. Conversely, the solid and alveolar variants are both characterized by classic ILC cells (small, regular sized, and lacking cohesion) that are arranged in sheets (solid type) or in aggregates of at least 20 cells (alveolar type) rather than in single cords of cells [1]. Furthermore, around 5\% of all invasive breast tumours exhibit mixed features of both ductal and lobular differentiation $[2,3]$.

Lobular intraepithelial neoplasia (LIN) frequently coexists with classical ILC in $90 \%$ of cases [4]. 95\% of ILC express oestrogen receptor (ER), and 60-70\% express progesterone receptor (PR) [57]. The proliferation index (measured by Ki67 staining) is usually low, and this likely contributes to reduced response to chemotherapy relative to patients with invasive ductal carcinoma (IDC) [1].

By virtue of their distinctive growth pattern and biology, lobular carcinomas have a substantially increased propensity for multifocal and multicentric distribution and for bilaterality [8-10]. Although associated with a less aggressive phenotype, this is offset by being more difficult to detect early, either by clinical examination or radiological imaging [6]. ILC do not usually form distinct mass lesions, making early diagnosis challenging $[8,11]$ and breast conservation approaches more difficult.

Mammography [8, 12, 13], ultrasonography [14], and magnetic resonance imaging (MRI) [15] have limitations in the diagnosis of ILC [16]. Although MRI has been found to have a higher sensitivity than mammography $[15,17]$, its sensitivity is still lower than for

\section{KARGER}

(c) 2017 S. Karger GmbH, Freiburg 
other invasive cancers. This may be attributed to the fact that ILC shows only subtle enhancement and its distribution mimics that of normal breast parenchyma [16].

Given their unique, albeit clinically and radiologically unpredictable, characteristics, there is a continuous debate regarding the optimal choice of surgery in ILC. There has been an increasing trend in recent years towards breast conserving surgery (BCS) as opposed to mastectomy. However, because of the infiltrative growth pattern and frequent discontinuities, there is a higher incidence of resection margin involvement in ILC than for IDC [12].

The purpose of this study was to evaluate the correlation between radiological and histological parameters in ILC and to identify predictive factors on core biopsies which may impact radiological appearance and final excision histology. The ultimate aim is to identify objective parameters to facilitate decision-making as to the best surgical option on an individual basis.

\section{Patients and Methods}

All biopsy-proven, newly diagnosed cases of ILC over 2 years (2013-2014) were included in the study. The cases were retrieved by SNOMED from the histopathology database at University Hospital Waterford (UHW), Ireland.

Radiological evaluation by mammography, breast ultrasonography, and MRI was performed in all the cases prior to surgical intervention. The breast density as assessed by mammography was recorded. The imaging modality that contributed to determining the clinical tumour size was also documented. A breast MRI was performed to identify the local disease extent and disease focality (unifocal vs. multifocal), and in some cases to assess for bilateral involvement.

A retrospective review of all the histological slides for all the cases that underwent surgical treatment was performed. The corresponding core biopsy, initial surgical resection, and subsequent surgical resections (where applicable) were reviewed for each case by a consultant histopathologist with special interest in breast pathology and a specialist histopathology trainee. The pattern of invasion was documented and comparisons were made between the core biopsy and resection specimens. The E-cadherin, ER, and PR status were documented.

The radiological tumour size and tumour focality were correlated with the histological findings on the resection specimens. The radiological and histological tumour sizes were considered discordant when the difference in size was more than $5 \mathrm{~mm}$. The margin status was assessed in each individual case, and subsequent surgical treatment documented. A positive margin was defined as invasive tumour present on ink or lying less than $1 \mathrm{~mm}$ from the inked surgical resection margin.

The correlation between radiology/histology discordance and menopausal status, breast density, pattern of invasion, presence of LIN, E-cadherin expression, ER/PR status, and Ki67 was determined.

Data were analysed using Microsoft Excel (Microsoft, Redmond, WA, USA) and SPSS v.20 (IBM Corp., Armonk, NY, USA). Statistical analysis was performed, where appropriate, using Fisher's exact test with a significance level of $<0.05$, and ANOVA with a confidence interval of $95 \%$.

\section{Results}

A total of 75 new cases of ILC were diagnosed at UHW between January 2013 and December 2014. The median age at diagnosis was 68 years (range $37-97$ years). $84 \%$ of cases occurred in postmenopausal women.
Almost two-thirds of the patients $(64 \%, \mathrm{n}=48)$ underwent surgery, while $36 \%(n=27)$ did not have any form of surgery. The latter were either unsuitable for surgical intervention due to metastatic disease at presentation or other comorbidities. Of those that underwent surgery, $75 \%(\mathrm{n}=36)$ had 1 operation, while $20.8 \%$ $(\mathrm{n}=10)$ and $4.2 \%(\mathrm{n}=2)$ underwent 2 or more than 2 operations, respectively. $62.5 \%(\mathrm{n}=30)$ had mastectomy as the initial curative surgery, while $37.5 \%(\mathrm{n}=18)$ underwent BCS. Of those who had BCS, $61.1 \%(n=11)$ had positive margins, of which $38.9 \%(n=7)$ had additional surgery. 4 patients received neoadjuvant therapy prior to surgery.

Full radiological assessment (mammography, breast ultrasound, and MRI) was not able to identify the presence of tumour in $8.3 \%$ $(n=4 / 48)$ of patients. Tumour was suspected clinically in these patients, and clinical core biopsies showed ILC. Tumour was identified on MRI in 92\% ( $\mathrm{n}=44 / 48$ ) of cases, by ultrasonography in $89.6 \%(n=43 / 48)$, and by mammography in only $73 \%(n=35 / 48)$.

Patients who received neoadjuvant therapy $(n=4)$ were excluded from the analysis of radiology-histology correlations, as neoadjuvant therapy precludes a correlation between pre-treatment radiological size and post-treatment histological size. Similarly, patients with negative imaging $(n=4)$ were also excluded. 40 patients had diagnostic radiology and underwent surgery without receiving neoadjuvant therapy.

ILC was multifocal in 45\% ( $\mathrm{n}=18 / 40)$ as confirmed by histology. There was concordance between radiological and histological tumour focality in almost two-thirds of cases $(65 \%, n=26 / 40)$. There was discordance in $35 \%(\mathrm{n}=14 / 40)$. In 9 cases, radiology identified a unifocal tumour, whereas it was multifocal on final excision histology.

Tumour size ranged from $1.5-\mathrm{mm}$ foci in multifocal disease to $>100 \mathrm{~mm}$. There was concordance between radiological and final histological tumour size in $60 \%(\mathrm{n}=24)$. In the concordant cases, the variation in tumour size was $5 \mathrm{~mm}$ or less. In $40 \%(\mathrm{n}=16)$ of cases, the discordance between radiological and histological tumour size exceeded $5 \mathrm{~mm}$, with a range of 6-78 $\mathrm{mm}$. In the 16 discordant cases, radiology underestimated the tumour size in $75 \%$ ( $\mathrm{n}=12$ ) of cases, and overestimated it in a smaller proportion of cases $(25 \%, \mathrm{n}=4)$.

On mammography, breast density was dense, moderately dense, and fatty (low density) in $18.6,74$, and $7.4 \%$, respectively. In 3 cases, breast density was difficult to assess due to scarring following previous surgery for benign breast disease in 2 cases and breast implants in a third case. The correlation between menopausal status and breast density was not statistically significant. In addition, the correlation between age at diagnosis, menopausal status, breast density, and radiology/histology discordance was also not statistically significant using ANOVA with a confidence interval of $95 \%$.

The pattern of invasion was predominantly classical in $47.5 \%$ (n = 19), trabecular in 35\% ( $\mathrm{n}=14)$, alveolar in $12.5 \%(\mathrm{n}=5)$, and solid in $5 \%(\mathrm{n}=2)$ (fig. 1). The correlation between histological pattern of invasion and radiology/histology discordance was not statistically significant using Fisher's exact test (2-tailed $\mathrm{p}$ value $=$ 0.8676) (table 1). 

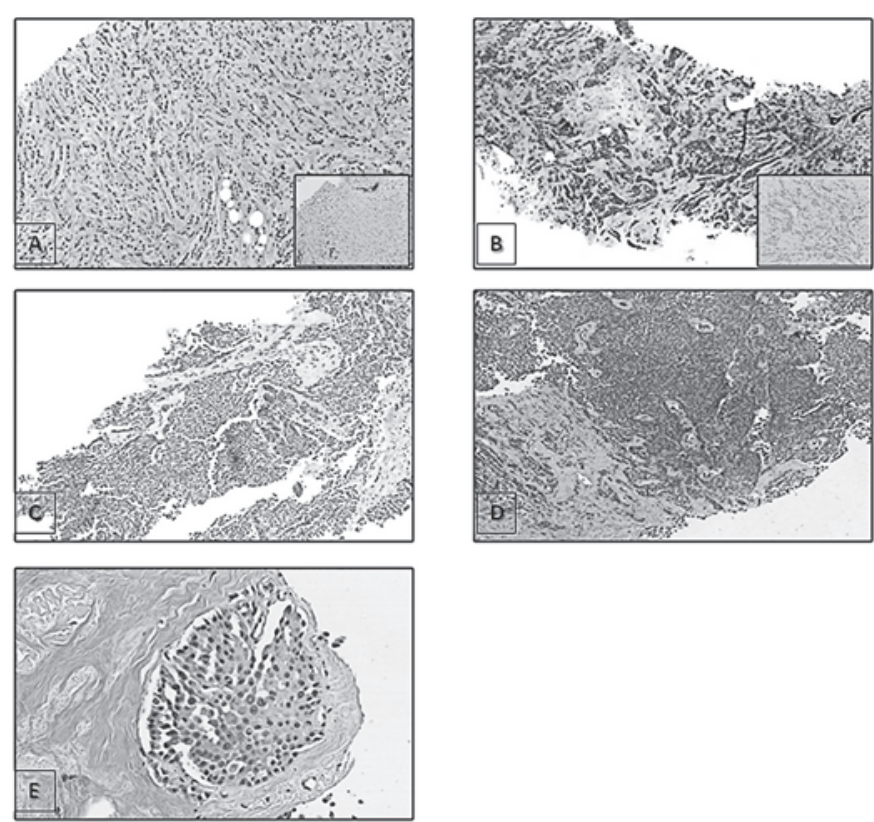

Fig. 1. Invasive lobular carcinoma, haematoxylin \& eosin stain, and E-cadherin immunohistochemistry (inset). A Classical, B trabecular, $\mathbf{C}$ alveolar, and D solid growth pattern; E lobular intraepithelial neoplasia (LIN).

E-cadherin immunohistochemistry was performed in all cases of newly diagnosed ILC. E-cadherin was negative in $81.3 \%(\mathrm{n}=61)$ and positive in $17.3 \%(\mathrm{n}=13)$, and there was mixed staining in $1.3 \%(\mathrm{n}=1)$. The correlation between E-cadherin status and radiology/histology discordance was significant using Fisher's exact test $(2$-tailed $\mathrm{p}$ value $=0.0229)$.

LIN was present in core biopsy specimens in $22.5 \%(n=9)$. Radiology/histology discordance was 44.4 and $38.7 \%$ for cases with and without LIN, respectively. However, this association was not statistically significant using Fisher's exact test (2-tailed $\mathrm{p}$ value $=$ 0.4777).

A total of $75 \%$ were positive for both $\mathrm{ER}$ and $\mathrm{PR}(\mathrm{ER}+\mathrm{PR}+)$, while the remainder were positive for ER and negative for $\mathrm{PR}$ (ER+/PR-). Radiology/histology discordance was 36.7 and 50\% for cases which were $\mathrm{ER}+/ \mathrm{PR}+$ and $\mathrm{ER}+/ \mathrm{PR}-$, respectively. However, the association between ER/PR status and radiology/histology discordance was not statistically significant using Fisher's exact test $(2$-tailed $\mathrm{p}$ value $=0.0661)$.

The Ki67 proliferation index was low $(<10 \%)$ to borderline $(10$ $20 \%$ ) in $87.2 \%$ of cases. Ki67 was not done in 1 case. The correlation between Ki67 and radiology/histology discordance was not statistically significant using ANOVA with a confidence interval of $95 \%$.

\section{Discussion}

Morphologically, all 75 newly diagnosed cases had a lobular growth pattern/architecture on haematoxylin \& eosin (H\&E) staining. Of these, only 48 patients underwent surgery. Overall, 25\% $(n=12)$ of all patients undergoing surgical treatment for ILC had 2 or more operations. Indeed, the diminished fibrotic reaction pre-
Table 1. Correlation between pattern of invasion and radiology/histology discordance

\begin{tabular}{lll}
\hline Predominant pattern & Patients, n (\%) & Discordance, (n) \% \\
\hline Classical & $19(47.5)$ & $8(42.1)$ \\
Trabecular & $14(35)$ & $6(42.8)$ \\
Alveolar & $5(12.5)$ & $1(20)$ \\
Solid & $2(5)$ & $1(50)$ \\
\hline
\end{tabular}

sent in ILC makes it difficult for surgeons to determine the gross extent of the disease during surgery. When this is coupled with the decreased sensitivity of imaging studies, it can be very challenging to achieve tumour-free margins after a limited excision.

Detailed histological analysis was feasible only for the cases where a surgical resection specimen was available. In these cases, an in-depth evaluation of the radiological findings was performed. MRI was able to detect the presence of tumour in $92 \%$ of these cases, making it the most sensitive imaging modality. Breast ultrasound also had a high detection rate of $89.6 \%$, while mammography detected only $73 \%$. However, in 4 patients all 3 imaging modalities failed to identify the presence of tumour. Despite the high detection rate, the rate of discordance between radiology and histology in detecting tumour focality was still high at $35 \%$ while the radiology/histology discordance with regard to tumour size was $40 \%$. Tumour size was underestimated radiologically in 12 cases, while it was overestimated in 4 cases. In the discordant cases, the difference in tumour size ranged from 6 to $78 \mathrm{~mm}$. This can have a considerable impact on T staging of ILC. This discordance was not unexpected, as other studies showed the sensitivities of mammography, sonography, and MRI for ILC to be 79,68 , and $83 \%$, respectively [18]. Another study looking at histologic subtype and imaging highlighted that the greatest differences between ultrasound and pathology measurements were observed in lobular carcinoma [19]; however, this study involved a smaller number of cases. Interestingly, breast density as assessed by mammography did not have a statistically significant impact on radiology/histology discordance. Of note, breast density did not correlate with menopausal status and age at diagnosis, and these parameters seem to be independent of each other. This was an unexpected finding, as the assumption was that the density of breast tissue decreases with age, making imaging more sensitive in detecting ILC with increasing age and resulting in a reduction in discordance rate. However, this was not the case in our study.

Histological findings were similar between core biopsies and subsequent resection specimens. The predominant pattern of invasion was 'classical' in almost half the cases (47.5\%), followed by trabecular and a smaller percentage of alveolar and solid patterns (5\%). The correlation between pattern of invasion and radiology/histology discordance was not significant. Discordance between imaging and histology was present even in ILC with alveolar and solid growth patterns. A possible explanation could be the presence of focal areas with classical growth pattern, which may contribute to the discordance. An illustrative example of this radiology/histology discordance is given in figure 2. In our opinion, on retrospective review of both 

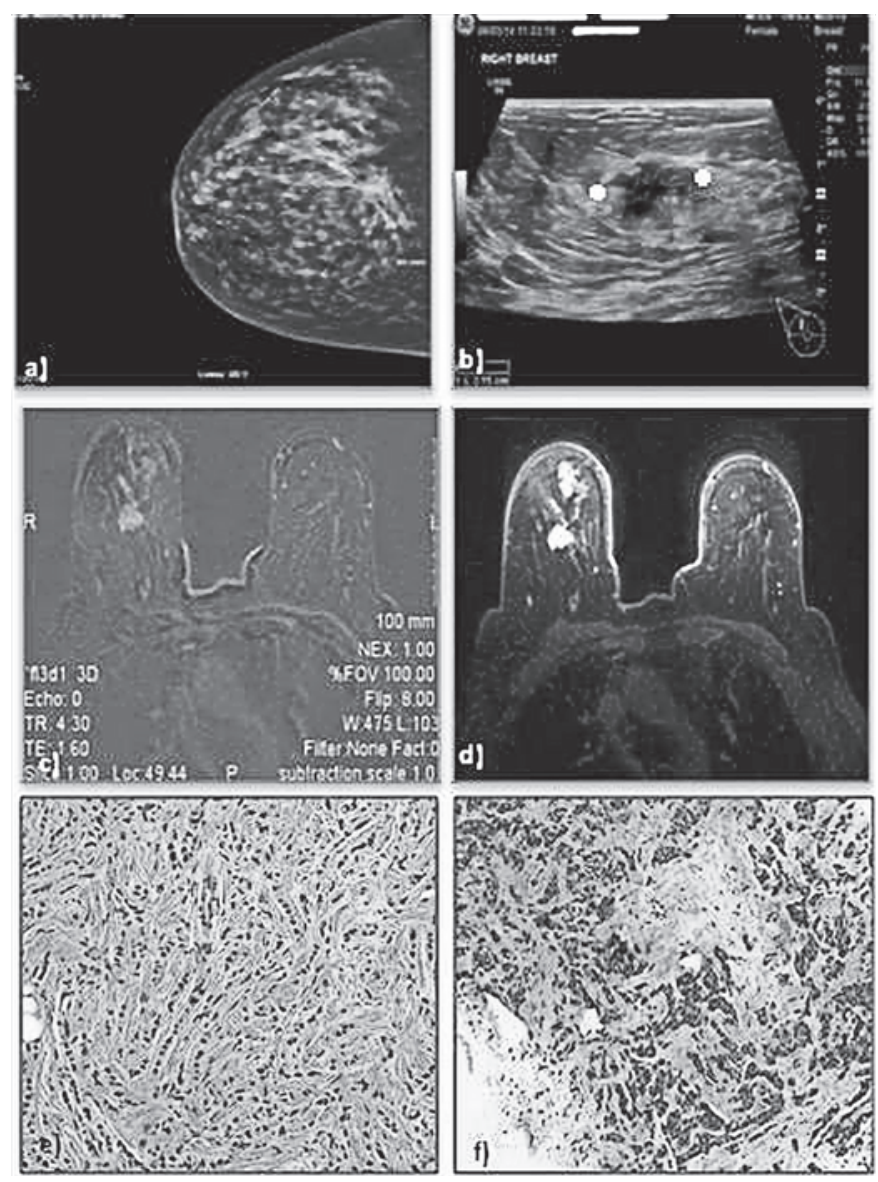

Fig. 2. a Mammography: both breasts are dense; new area of slight distortion present at 12 o'clock in the right breast. b Ultrasound: a $2.2-\mathrm{cm}$ irregular hypoechoic lesion was identified. c, d Magnetic resonance imaging: 2-cm enhancing lesion consistent with the known neoplasm; in addition, there is a linear area of enhancement between this lesion and the right nipple-areolar complex; further evaluation needed if patient opted for breast conservation. e, f Histology: invasive lobular carcinoma, mixed classical and trabecular growth pattern, more than $100 \mathrm{~mm}$ with multifocal distribution.

the imaging and histology, it is most likely that the 'trabecular' pattern contributed to the mammographically and ultrasound-detected distortion/lesion, while the linear enhancement on MRI correlates with the 'classical' pattern which is non-mass-forming.

The vast majority of cases ( $81.3 \%)$ were E-cadherin negative, while $17.3 \%$ were E-cadherin positive. E-cadherin expression has become an important diagnostic feature of LIN and ILC [20]; however, it is important to remember that approximately $10 \%$ of ILC still express E-cadherin [21, 22] either with normal membrane localisation or aberrantly distributed as fragmented membrane and/ or cytoplasmic staining. The E-cadherin-catenin complex may be dysfunctional in these cases due to the presence of $\mathrm{CDH} 1$ gene mutation or aberrant/loss of expression of the catenin binding proteins [22], which may be detected using $\beta$-catenin and p120catenin immunohistochemistry. However, a diagnosis of LIN or ILC based on morphological and cytological criteria should therefore not be reclassified as ductal carcinoma in situ or IDC of no special type (IDC-NST) based on the status of these immunohistochemical markers [20]. At our institution, management of lobular carcinoma is based on the morphological (H\&E diagnosis) rather than the E-cadherin staining of the tumour. Controversially, an interesting finding in our study was the statistically significant association between E-cadherin immunostaining and radiology/ histology concordance. Half of these cases had a 'classical' growth pattern, while the remainder had mixed 'classical, trabecular, and alveolar' growth patterns. LIN was present in half the cases that were E-cadherin positive. The cases which were E-cadherin-positive were more likely to have concordant radiological and histological size, suggesting that positive E-cadherin may pertain to more localized tumour infiltration despite the lobular morphology.

LIN was present on core biopsy in $22.5 \%$. However, it was not a helpful predictive parameter for radiology/histology concordance. $75 \%$ of cases were positive for both ER and PR, and $87.2 \%$ had a low to borderline proliferation index as assessed by Ki67. Overall, the biological profile of ILC did not have a significant correlation with radiology/histology discordance.

The main limitation of this study is the low number of cases of surgically treated ILC; however, it does provide an insight into the behaviour of these tumours. It highlights the importance of E-cadherin staining, even in this limited sample. There has been general acceptance that morphology precedes E-cadherin immunostaining in predicting tumour behaviour; however, our study sheds some doubt on this. The pattern of invasion in ILC does not seem to play a role in improved radiological correlation; however, a large series of alveolar and solid patterns may prove otherwise. The main difficulty in identifying this subset is that the pattern of invasion of ILC is not routinely reported on core biopsy specimens, which makes case selection a cumbersome process. The best way to overcome this is to carry out a prospective study recording pattern of invasion on core biopsy and correlating these with the final histology. A larger series may be able to identify a role for ER/PR hormonal status and Ki67; however, given that almost all ILC are ER-positive and the majority are PR-positive, this is unlikely.

\section{Conclusion}

The majority of ILC are characterized by a subtle infiltrative pattern which makes it difficult to radiologically estimate tumour focality and size. Our study highlights that radiological assessment underestimates tumour size in more than one-third of cases. This impacts the decision for curative surgery. Despite the heterogeneity of ILC, histological and biological factors, with the exception of Ecadherin expression, do not seem to play a significant role in the discordance between radiology and histology. More sensitive imaging techniques may allow for a more accurate pre-operative assessment in BCS for ILC and increase the concordance between radiology and histology.

\section{Disclosure Statement}

The authors declare no conflicts of interest and no sources of funding or sponsorship. 


\section{References}

1 McCart Reed AE, Kutasovic JR, Lakhani SR, Simpson PT: Invasive lobular carcinoma of the breast: morphol ogy, biomarkers and 'omics. Breast Cancer Res 2015 17:12.

2 Lakhani SR, Ellis IO, Schnitt SJ, Tan PH, van de Vijver MJ: WHO Classification of Tumours of the Breast, 4th ed. Lyon, IARC, 2012.

3 Rakha EA, Gill MS, El-Sayed ME, Khan MM, Hodi Z, Blamey RW, Evans AJ, Lee AH, Ellis IO: The biological and clinical characteristics of breast carcinoma with mixed ductal and lobular morphology. Breast Cancer Res Treat 2009;114:243-250.

4 Abdel-Fatah TM, Powe DG, Hodi Z, Lee AH, ReisFilho JS, Ellis IO: High frequency of coexistence of columnar cell lesions, lobular neoplasia, and low grade ductal carcinoma in situ with invasive tubular carcinoma and invasive lobular carcinoma. Am J Surg Pathol 2007;31:417-426.

5 Rakha EA, El-Sayed ME, Powe DG, Green AR Habashy H, Grainge MJ, Robertson JF, BlameyR, Gee J, Nicholson RI, Lee AH, Ellis IO: Invasive lobular carcinoma of the breast: response to hormonal therapy and outcomes. Eur J Cancer 2008;44:73-83.

6 Arpino G, Bardou VJ, Clark GM, Elledge RM: Infiltrating lobular carcinoma of the breast: tumor characteristics and clinical outcome. Breast Cancer Res 2004;6: R149-156.

7 Sastre-Garau X, Jouve M, Asselain B, Vincent-Salomon A, Beuzeboc P, Dorval T, Durand JC, Fourquet A Pouillart P: Infiltrating lobular carcinoma of the breast. Clinicopathologic analysis of 975 cases with reference to data on conservative therapy and metastatic patterns. Cancer 1996;77:113-120.
8 Krecke KN, Gisvold JJ: Invasive lobular carcinoma of the breast: mammographic findings and extent of disease at diagnosis in 184 patients. AJR Am J Roentgenol 1993;161:957-960.

9 Cornford EJ, Wilson AR, Athanassiou E, Galea M, Ellis IO, Elston CW, Blamey RW: Mammographic features of invasive lobular and invasive ductal carcinoma of the breast: a comparative analysis. Br J Radiol 1995;68: 450-453.

10 Lesser ML, Rosen PP, Kinne DW: Multicentricity and bilaterality in invasive breast carcinoma. Surgery 1982; 91:234-240.

11 Yeatman TJ, Cantor AB, Smith TJ, Smith SK, Reintgen DS, Miller MS, Ku NN, Baekey PA, Cox CE: Tumor biology of infiltrating lobular carcinoma. Implications for management. Ann Surg 1995;222:549-559, discussion 559-561.

12 Holland R, Hendriks JHCL, Mravunac M: Mammographically occult breast cancer: a pathologic and radiologic study. Cancer 1983;52:1810-1819.

13 Sickles EA: The subtle and atypical mammographic features of invasive lobular carcinoma. Radiology 1991;153:25-26.

14 Selinko VL, Middleton LP, Dempsey PJ: Role of sonography in diagnosing and staging invasive lobular carcinoma. J Clin Ultrasound 2004;32:323-332.

15 Boetes C, Veltman J, van Die L, Bult P, Wobbes T, Barentsz JO: The role of MRI in invasive lobular carcinoma. Breast Cancer Res Treat 2004;86:31-37.

16 Kopans DB: Malignant lesions of the lobule; in Kopans DB (ed): Breast Imaging. Baltimore, MD, Lippincott Williams \& Wilkins, 2007, pp. 866-870.
17 Mann RM, Hoogeveen YL, Blickman JG, Boetes C: MRI compared to conventional diagnostic work-up in the detection and evaluation of invasive lobular carcinoma of the breast: a review of existing literature. Breast Cancer Res Treat 2008;107:1-14.

18 Brem RF, Ioffe M, Rapelyea JA, Yost KG, Weigert JM, Bertrand ML, Stern LH: Invasive lobular carcinoma: detection with mammography, sonography, MRI, and breast-specific gamma imaging. AJR Am J Roentgenol 2009;192:379-383.

19 Pritt B, Ashikaga T, Oppenheimer RG, Weaver DL: Influence of breast cancer histology on the relationship between ultrasound and pathology tumor size measurements. Mod Pathol 2004;17:905-910.

20 Dabbs DJ, Schnitt SJ, Geyer FC, Weigelt B, Baehner FL, Decker T, Eusebi V, Fox SB, Ichihara S, Lakhani SR, Palacios J, Rakha E, Richardson AL, Schmitt FC, Tan PH, Tse GM, Vincent-Salomon A, Ellis IO, Badve S, Reis-Filho JS: Lobular neoplasia of the breast revisited with emphasis on the role of E-cadherin immunohistochemistry. Am J Surg Pathol 2013;37:e1-11.

21 Rakha EA, Ellis IO: Lobular breast carcinoma and its variants. Semin Diagn Pathol 2010;27:49-61.

22 Da Silva L, Parry S, Reid L, Keith P, Waddell N, Kossai M, Clarke C, Lakhani SR, Simpson PT: Aberrant expression of E-cadherin in lobular carcinomas of the breast. Am J Surg Pathol 2008;32:773-783. 